\title{
The BP network Design of MPPT with Photovoltaic Power System
}

\author{
Huaizhong Chen, Xiaoliang Wu, Tao Jin \\ Zhejiang Industry Polytechnic College, Shaoxing, China \\ Chz702@163.com
}

Keywords: photovoltaic power system; BP neural; network; MPPT; algorithm

\begin{abstract}
Photovoltaic power system has bigger time-vary nature, so it is difficult to build accurate mathematic model. BP neural network has the capability of expression nonlinearity and also has the self study and adaptive function。BP neural network control makes full use of neural network approximation capability, and with better control in resolving the highly nonlinear seriously uncertain systems. Simulated result indicates this control is able to make system reach satisfied control effect, accurately track the maximum power point of PV cells.
\end{abstract}

\section{Introduction}

Photovoltaic power generation system is a device that can directly convert solar energy into electrical energy. One voltage output characteristic of photovoltaic cell is nonlinear, and its output characteristics are influenced by the factors such as sunshine, temperature and so on. In addition, photovoltaic efficiency is very low, the price is expensive, large initial investment, therefore, it is necessary to adopt the maximum power tracking control to improve the efficiency of the PV system. The commonly used maximum power point tracking method with perturbation and observation method, constant voltage method, incremental conductance method and optimal gradient method etc.. These methods are difficult to realize control. This paper presents a BP neural network control method, the simulation results show that this method can achieve better control performance[1].

\section{Photovoltaic MPPT control system}

2.1 The Equivalent Model of Photovoltaic Cell. In Fig.1, it is composed of ideal current source Is, anti-parallel diode D, series resistance Rs and the parallel resistance Rsh. Among them, the Is value is equal to the value of the cells' short-circuit current; its size reflects the sunlight intensity of the environment where the photovoltaic cell is located.

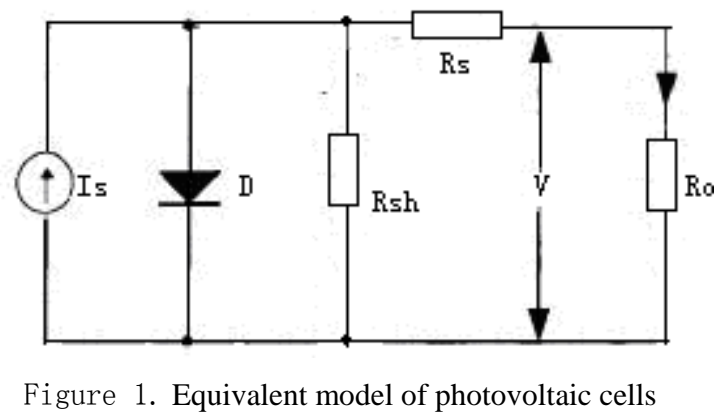

The formula below is a equation between the characteristics of I and $\mathrm{V}$ in photovoltaic cell:

$$
I=I_{s}-I_{o}\left[\exp \left[\frac{q\left(V+R_{S} I\right)}{A K T}\right]-1\right]-\frac{q\left(V+R_{S} I\right)}{R_{s h}}
$$


Under ideal situation, Rs can be approximate to zero, and Rsh is approximate to infinity, then the formula above can be simplified as:

$$
I=I_{s}-I_{o}\left[\exp \left[\frac{q\left(V+R_{S} I\right)}{A K T}\right]-1\right]
$$

In the formula, I is working current; $\mathrm{I}_{\mathrm{o}}$ is reverse saturation current; $\mathrm{V}$ is the output voltage of cell, the others are content. Thus, the output power of photovoltaic cell is:

$$
P=I_{s} \cdot V-I_{o} \cdot V\left\{\exp \left[\frac{q}{A K T}\right]-1\right\}
$$

It shows that the output power of photovoltaic cell is in a nonlinear function between the sunlight intensity and temperature, but it is in a proportional relation with the current and voltage.

2.2 MPPT control. The maximum power point tracking is that the controller can generate voltage real-time detection of solar panels, and track the appropriate voltage and current value, the system with the highest output efficiency. Fig.2 is volt battery characteristic curve[2].

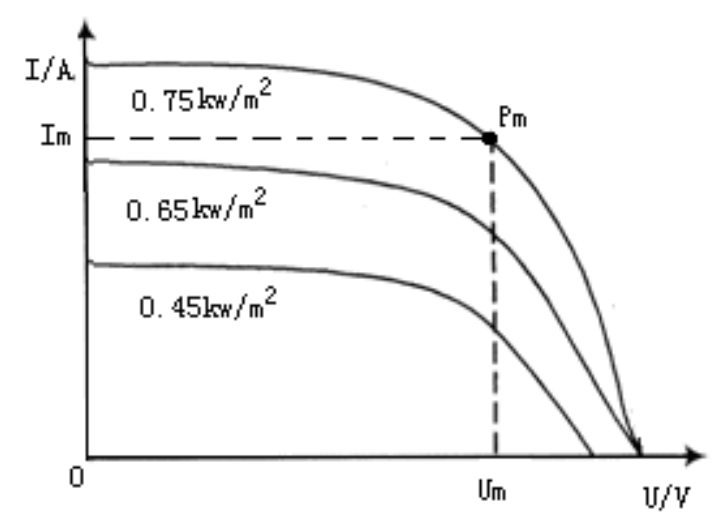

Figure 2. Characteristic curve when $\mathrm{T}=250$

From above picture, we can see that, in a certain temperature and light intensities, different solar light intensity curve can find a $\mathrm{P}_{\mathrm{m}}$ maximum power output point, assuming a solar photovoltaic battery work almost always work hours at the maximum power point $\mathrm{P}_{\mathrm{m}}$, it can greatly improve the efficiency of the solar photovoltaic energy conversion efficiency rise, how to use the best way to find the maximum power point, and work in the vicinity of the maximum power, maximum power point automatic optimization.

\section{BP neural network based on control algorithm structure}

3.1 Network control structure. Fig.3 presents a BP network, constituting of input nodes, output nodes, and nodes of multiple hidden layers.

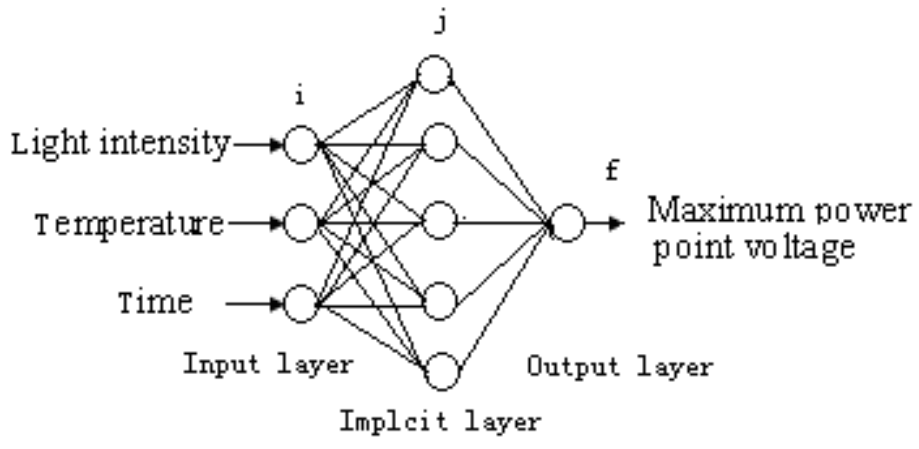

Figure 3. Network control structure 
The learning process of BP neural network has two phases: In the first phase information propagates forward from input of signals into input layer, to be processed by hidden layers one by one, and then to be calculated by the output layer to output values. The second phase is the process of error back propagation. When values output are not as expected, then the difference/error between the actual output and the expected output will be calculated out layer by layer and the weight will be updated according to this error[3]. As the light intensity, temperature and time. The output variable is only one, that is the maximum power point voltage. Determination of the hidden layer nodes have certain principles, if the node number is too small, small to create a network mapping complex, the network learning and training effect is poor, fault tolerance. Small ideal; when the number of nodes too much, it will lead to the network is too large, complex structure, learning. Time is too long, even result in small convergence[4].

\subsection{The forward propagation algorithm of neural network}

1) Input of the input layer is:

$$
O_{i}^{(1)}=x(j)
$$

2) Input and output of hidden layer are:

$$
\begin{gathered}
n e t_{i}^{(2)}(k)=\sum_{j=0}^{M} w_{i j}^{(2)} O_{j}^{(1)} \\
O_{i}^{(2)}(k)=f\left(\text { net }_{i}^{(2)}(k)\right)
\end{gathered}
$$

Of which $w_{i j}$ is the weight of hidden layer, and corner labels 1), 2), and 3) represent input layer, hidden layer, and output layer. To use the unipolar sigmoid function of hidden layer:

$$
\begin{gathered}
f(x)=\tanh (x)=\frac{1}{1+e^{-x}} \\
O_{i}^{(2)}(k)=\frac{1}{1+\exp \left(n e t_{i}^{(2)}-\theta_{j}^{(2)}\right)}
\end{gathered}
$$

Of which $\theta_{j}^{(2)}$ is the threshold of No.j neuron of the second layer.

3) The empirical formula for determining the number of nodes in the hidden layer is much, the common method are:

$$
\begin{aligned}
& m=\sqrt{n+l}+C \\
& m=\log _{2}^{n} \\
& m=\sqrt{n}
\end{aligned}
$$

Where $\mathrm{m}$ is the number of hidden layer nodes; $\mathrm{N}$ is input nodes; 1 is output nodes; $\mathrm{C}$ is constant between 1-10. In the photovoltaic power generation in the MPPT algorithm, $n$ is 3,1 is 1 .

\section{Simulation}

We Sample light intensity, temperature data in one day $24 \mathrm{~h}$, through the BP neural network structure for learning and training. In order to avoid errors fall into the local minimum of the error surface, we determine the maximum number of training of 800 , and fix target function are square error is 0.01 . Training function uses trainlm algorithm simulation test. The simulation waveforms are shown in fig. 4 . 


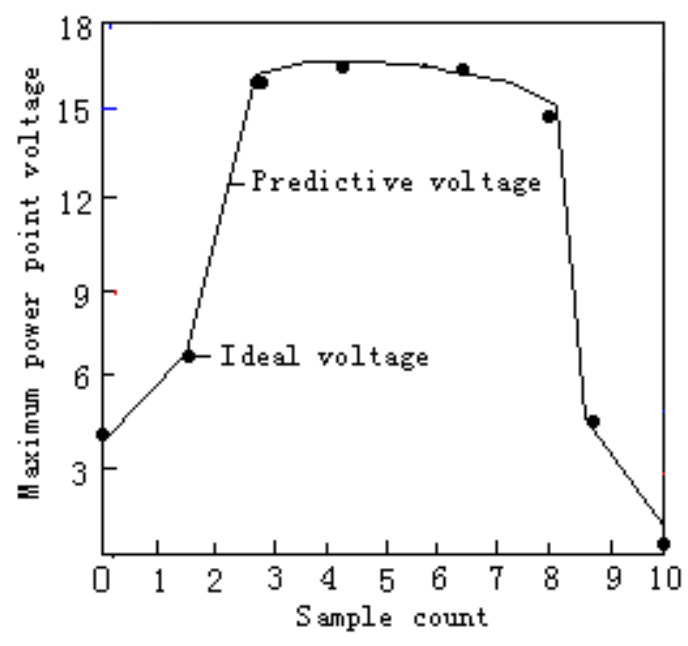

Figure 4. Maximum power point voltage prediction curve

Through analysis on the above control curve and data results, the following conclusion can be obtained: when BP neural network algorithm is applied to control system, the control system transition time is short and is able to reach steady state much faster, it still can reach desired control effect under the condition with interference, and it has stronger adaptability and robustness. It can be seen that the trainlm algorithm can better track the maximum power point voltage[5].

\section{Conclusion}

Based on the research of the maximum power output on the algorithm for photovoltaic power, this paper apply BP neural network control to photovoltaic cell MPPT. The simulation results show that compared with the conventional MPPT algorithm, the neural network control technique In the case of temperature, light intensity and other external environmental changes, the higher nonlinear mapping capability and fault tolerance, and the computation speed is obviously faster than other methods. The performance improves the efficiency, and has better control precision and stability.

\section{Acknowledgment}

This work is supported by scientific research funded project of Science Technology Department of Zhejiang Province (2015C31128).

\section{References}

[1]FURNABASHIT, KOYANAGIK, YOKOYAMAR. 2003.A Review of Islanding Detection Methods for Distributed Resources. Power Tech Conference Proceedings, IEEE Bologna, 30( 2): 1-6

[2]CHEN Yang. 2004.A Cost Effective Single Stage Inverter With Maximum Power Point Tracking. IEEE Transactions on Power Ele-tronics, 19( 5): 1289-1294

[3]Tang CC, Ho W K, Cao L S. 2004.A Comparison of Two Design Methods for PID Controllers. ISA TRANS, 9( 1):2-33.

[4]Eker.lyas.2003.Operation and control of water supply system, ISA Transactions, July, 2(3):461-473.

[5]B. Barak and M. Mahmoody-Ghidary.2007.Lower bounds of signatures from symmetric primitives, In 48th Annals Symposium on Foundations of Computer Science, 290(1-2): 680-688. 